relapse compared with those treated with lowdose cytarabine (hazard ratio 0.28, $P=0.002$ ). In the subgroup of patients without RAS mutations, those who received high-dose cytarabine had a lower risk of relapse than patients treated with low-dose cytarabine (hazard ratio 0.67 , $P=0.044)$ but this benefit was smaller than for RAS-mutation carriers.

Original article Neubauer A et al. (2008) Patients with acute myeloid leukemia and RAS mutations benefit most from postremission high-dose cytarabine: a Cancer and Leukemia Group B study. J Clin Oncol 26: 4603-4609

\section{Erlotinib prolongs time to progression in patients with EGFR-positive NSCLC}

In 2005, the TRIBUTE trial demonstrated that addition of erlotinib to chemotherapy did not improve overall survival or time to progression in patients with advanced non-small-cell lung cancer (NSCLC). On the basis that increased EGFR copy number is associated with improved overall survival and time to progression after treatment with anti-EGFR agents, Hirsch et al. examined whether EGFR copy numbers could predict outcome in patients with NSCLC.

Fluorescent in situ hybridization was used to assess EGFR gene amplification (i.e. increased copy numbers) in 275 tumor samples obtained from TRIBUTE participants. Of these samples, 100 showed amplification of EGFR and 145 did not (EGFR+ ${ }^{+}$and $E G F R^{-}$, respectively). For patients whose tumors were $E G F R^{+}$, the median time to progression rates were 6.3 months and 5.8 months for patients who received chemotherapy plus erlotinib, and for those who received chemotherapy plus placebo $(P=0.0430)$. The median times to progression for $E G F R^{-}$patients were 4.6 months and 6.0 months for these groups, respectively $(P=0.0895)$. The objective response rate in patients whose tumors were EGFR ${ }^{+}$and who received erlotinib was particularly pronounced after completion of chemotherapy, which suggests that erlotinib could offer benefits as maintenance therapy. No significant effect of erlotinib on the objective response rate was seen in $E G F R^{-}$patients.

Although EGFR amplification does not correlate with improved survival, these results suggest that erlotinib prolongs time to progression in patients with NSCLC whose tumors show increased copy numbers of EGFR.

Original article Hirsch FR et al. (2008) Fluoresence in situ hybridization subgroup analysis of TRIBUTE, a phase III trial of erlotinib plus carboplatin and paclitaxel in non-small cell lung cancer. Clin Cancer Res 14: 6317-6323

\section{MPT improves progression-free survival in elderly patients with multiple myeloma}

In patients with multiple myeloma, the combination of melphalan, prednisone and thalidomide (MPT) has been shown to result in better response rates and progression-free survival than melphalan and prednisone (MP) alone. Palumbo and colleagues reported the updated results of a trial that compared oral MP and MPT treatments in elderly patients with multiple myeloma.

The study enrolled 331 patients with multiple myeloma aged $\geq 65$ years to receive either MP $(n=164)$ or MPT $(n=167)$. The primary end points were response rate and progressionfree survival, with overall survival as a secondary end point. With a median follow-up of 38.1 months, the median overall survival rates for patients in the MP and MPT groups, respectively, were 47.6 months and 45.0 months $(P=0.79)$. By contrast, the progression-free survival in these two groups were 14.5 months and 21.8 months, respectively $(P=0.0004)$. Compared with MP, MPT improved progression-free survival in patients $>75$ years, who had increased $\beta_{2}$-microglobulin levels, high International Staging System disease stage, anemia, renal insufficiency or bone-marrow plasmacytosis. Bortezomib or thalidomide administration as salvage therapy significantly improved survival after progression in patients who received MP at diagnosis $(P=0.0002)$, but not in patients who were treated with MPT.

This study showed that in elderly patients with multiple myeloma, oral MPT therapy improved response and progression-free survival rates but not overall survival, compared with MP therapy alone.

Original article Palumbo A et al. (2008) Oral melphalan, prednisone, and thalidomide in elderly patients with multiple myeloma: updated results of a randomized controlled trial. Blood 112: 3107-3114 